PROCEEDINGS OF THE

AMERICAN MATHEMATICAL SOCIETY

Volume 140, Number 5, May 2012, Pages 1633-1641

S 0002-9939(2011)11016-5

Article electronically published on August 19, 2011

\title{
A NOTE ON THE GEOMETRY OF PSEUDOCONVEX DOMAINS OF FINITE TYPE IN ALMOST COMPLEX MANIFOLDS
}

\author{
FLORIAN BERTRAND
}

(Communicated by Franc Forsternic)

\begin{abstract}
Let $D=\{\rho<0\}$ be a smooth domain of finite type in an almost complex manifold $(M, J)$ of real dimension four. We assume that the defining function $\rho$ is $J$-plurisubharmonic on a neighborhood of $\bar{D}$. We study the asymptotic behavior of pseudoholomorphic discs contained in the domain $D$.
\end{abstract}

\section{INTRODUCTION}

A well known problem is to determine which smooth domains in an almost complex manifold $(M, J)$ are locally complete hyperbolic in the sense of Kobayashi. It seems natural to make some curvature assumptions on such domains, as any $J$ pseudoconcavity boundary point is at finite Kobayashi distance to the interior of the domain [7. I. Graham [6] gave asymptotic estimates of the Kobayashi pseudometric for strictly pseudoconvex bounded domains into $\left(\mathbb{C}^{n}, J_{s t}\right)$ and proved the complete hyperbolicity of those domains. In case $(M, J)$ is any almost complex manifold, similar results were provided by S. Ivashkovich and J.-P. Rosay [7] and H. Gaussier and A. Sukhov [5].

The situation is far from being so clear when the domains are weakly pseudoconvex, as the geometry of their boundary is much more complicated. Also, the question of whether a smooth weakly pseudoconvex domain is locally complete hyperbolic or not is still open, even in $\left(\mathbb{C}^{2}, J_{s t}\right)$. However, D. Catlin 3 obtained local estimates similar to those obtained in [6] on smooth pseudoconvex domains of finite type in $\left(\mathbb{C}^{2}, J_{s t}\right)$, implying their local complete hyperbolicity (see also [1]).

In the present paper we study this question for smooth $J$-pseudoconvex domains of finite type in a four dimensional almost complex manifold. In [2, we described locally finite type domains $D=\{\rho<0\}$, where $\rho$ is a smooth defining function for $D$ and $J$-plurisubharmonic (see Proposition 2.6 in 2]). More precisely, if $D=$ $\{\rho<0\} \subset \mathbb{C}^{2}$ and if the origin $0 \in \partial D$ is of finite type $2 m$, we proved that there is a change of coordinates in a neighborhood of the origin such that the structure $J$

Received by the editors August 17, 2010 and, in revised form, January 11, 2011.

2000 Mathematics Subject Classification. Primary 32Q60, 32T25, 32T40, 32Q45, 32 Q65.

Key words and phrases. Almost complex structure, Kobayashi pseudometric, D'Angelo type.

(C)2011 American Mathematical Society

Reverts to public domain 28 years from publication 
and the function $\rho$ can be locally written as

$$
J=\left(\begin{array}{cccc}
a_{1} & b_{1} & 0 & 0 \\
c_{1} & -a_{1} & 0 & 0 \\
0 & 0 & a_{2} & b_{2} \\
0 & 0 & c_{2} & -a_{2}
\end{array}\right)=J_{s t}+O\left(\left|z_{2}\right|\right)
$$

and

$$
\rho=\Re e z_{2}+H_{2 m}\left(z_{1}, \overline{z_{1}}\right)+\widetilde{H}\left(z_{1}, z_{2}\right)+O\left(\left|z_{1}\right|^{2 m+1}+\left|z_{2}\right|\left|z_{1}\right|^{m}+\left|z_{2}\right|^{2}\right),
$$

where $H_{2 m}$ is a homogeneous polynomial of degree $2 m$, subharmonic which is not harmonic and $\widetilde{H}\left(z_{1}, z_{2}\right)=\Re e \sum_{k=1}^{m-1} \rho_{k} z_{1}^{k} z_{2}$.

In this paper, we suppose that the harmonic term $\widetilde{H}\left(z_{1}, z_{2}\right)$ in the above expression (0.2) is identically zero and we prove:

Theorem 0.1. Let $J$ be a smooth almost complex structure defined on $\mathbb{R}^{4}$. Let $D=\{\rho<0\}$ be a domain of finite type in $\left(\mathbb{R}^{4}, J\right)$, where $\rho$ is a smooth defining function of $D, J$-plurisubharmonic in a neighborhood of $\bar{D}$. We suppose that $J$ and $\rho$ satisfy respectively (0.1) and (0.2). Moreover, we assume that $\widetilde{H}\left(z_{1}, z_{2}\right)$ in (0.2) is identically zero. Then there exists a neighborhood $U$ of the origin for which 0 is at infinite distance from points in $D \cap U$.

The proof of this theorem was inspired by [7] and is based on the construction of good $J$-plurisubharmonic functions whose use is significant in almost complex manifolds.

\section{Preliminaries}

We denote by $\Delta$ the unit disc of $\mathbb{C}$ and by $\Delta_{r}$ the disc of $\mathbb{C}$ centered at the origin of radius $r>0$.

1.1. Almost complex manifolds and pseudoholomorphic discs. An almost complex structure $J$ on a real smooth manifold $M$ is a $(1,1)$ tensor field which satisfies $J^{2}=-I d$. We suppose that $J$ is smooth. The pair $(M, J)$ is called an almost complex manifold. We denote by $J_{\text {st }}$ the standard integrable structure on $\mathbb{C}^{n}$ for every $n$. A differentiable map $f:\left(M^{\prime}, J^{\prime}\right) \longrightarrow(M, J)$ between two almost complex manifolds is said to be $\left(J^{\prime}, J\right)$-holomorphic if $J(f(p)) \circ d_{p} f=d_{p} f \circ J^{\prime}(p)$ for every $p \in M^{\prime}$. In case $M^{\prime}=\Delta \subset \mathbb{C}$, such a map is called a pseudoholomorphic disc. If $f:(M, J) \longrightarrow M^{\prime}$ is a diffeomorphism, we define an almost complex structure, $f_{*} J$, on $M^{\prime}$ as the direct image of $J$ by $f$ :

$$
f_{*} J(q):=d_{f^{-1}(q)} f \circ J\left(f^{-1}(q)\right) \circ d_{q} f^{-1},
$$

for every $q \in M^{\prime}$.

The following lemma (see [5]) states that locally any almost complex manifold can be seen as the unit ball of $\mathbb{C}^{n}$ endowed with a small smooth perturbation of the standard integrable structure $J_{s t}$.

Lemma 1.1. Let $(M, J)$ be an almost complex manifold with $J$ of class $\mathcal{C}^{k}, k \geq 0$. Then for every point $p \in M$ and every $\lambda_{0}>0$ there exist a neighborhood $U$ of $p$ and a coordinate diffeomorphism $z: U \rightarrow \mathbb{B}$ centered a $p$ (i.e. $z(p)=0$ ) such that the direct image of $J$ satisfies $z_{*} J(0)=J_{\text {st }}$ and $\left\|z_{*}(J)-J_{s t}\right\|_{\mathcal{C}^{k}(\overline{\mathbb{B}})} \leq \lambda_{0}$. 
This is simply done by considering a local chart $z: U \rightarrow \mathbb{B}$ centered a $p$ (i.e. $z(p)=0$ ), composing it with a linear diffeomorphism to ensure $z_{*} J(0)=J_{s t}$ and dilating coordinates.

Therefore, let $J$ be an almost complex structure defined in a neighborhood $U$ of the origin in $\mathbb{R}^{2 n}$ and such that $J$ is sufficiently close to the standard structure in uniform norm on the closure $\bar{U}$ of $U$. The $J$-holomorphy equation for a pseudoholomorphic disc $u: \Delta \rightarrow U \subseteq \mathbb{R}^{2 n}$ is given by

$$
\frac{\partial u}{\partial y}-J(u) \frac{\partial u}{\partial x}=0
$$

According to [8], for every $p \in M$ there is a neighborhood $V$ of zero in $T_{p} M$ such that for every $v \in V$ there is a $J$-holomorphic disc $u$ satisfying $u(0)=p$ and $d_{0} u(\partial / \partial x)=v$.

1.2. Levi geometry. Let $\rho$ be a $\mathcal{C}^{2}$ real valued function on a smooth almost complex manifold $(M, J)$. We denote by $d_{J}^{c} \rho$ the differential form defined by

$$
d_{J}^{c} \rho(v):=-d \rho(J v),
$$

where $v$ is a section of TM. The Levi form of $\rho$ at a point $p \in M$ and a vector $v \in T_{p} M$ is defined by

$$
\mathcal{L}_{J} \rho(p, v):=d\left(d_{J}^{c} \rho\right)(p)(v, J(p) v)=d d_{J}^{c} \rho(p)(v, J(p) v) .
$$

The next proposition is useful in order to compute the Levi form (see [7]).

Proposition 1.2. Let $p \in M$ and $v \in T_{p} M$. Then

$$
\mathcal{L}_{J} \rho(p, v)=\Delta(\rho \circ u)(0),
$$

where $u: \Delta \rightarrow(M, J)$ is any $J$-holomorphic disc satisfying $u(0)=p$ and $d_{0} u\left(\partial / \partial_{x}\right)$ $=v$.

If $\mathcal{L}_{J} \rho(p, v) \geq 0$ for every $p \in M$ and every $v \in T_{p} M$, we say that $\rho$ is $J$ plurisubharmonic. It is a well known fact that $\rho$ is $J$-plurisubharmonic if and only if for every $J$-holomorphic disc $u: \Delta \rightarrow M, \rho \circ u$ is subharmonic (see [7]).

1.3. Pseudoconvex domains of finite type. In this section, we recall some facts about pseudoconvex domains of finite type in four dimensional almost complex manifolds (see [2] for more detailed facts).

Let $D=\{\rho<0\}$ be a smooth domain in $\mathbb{R}^{4}$. Assume that $\rho$ is $J$-plurisubharmonic on a neighborhood of $\bar{D}$ where the structure $J$ is defined on a fixed neighborhood $U$ of $\bar{D}$. Moreover, we suppose that the origin is a boundary point of $D$.

Definition 1.3. Let $u:(\Delta, 0) \rightarrow\left(\mathbb{R}^{4}, 0, J\right)$ be a $J$-holomorphic disc satisfying $u(0)=0$. The order of contact $\delta_{0}(\partial D, u)$ with $\partial D$ at the origin is the degree of the first term in the Taylor expansion of $\rho \circ u$. We denote by $\delta(u)$ the multiplicity of $u$ at the origin.

We now define the (D'Angelo) type and the regular type of the real hypersurface $\partial D$ at the origin. 


\section{Definition 1.4.}

(1) The (D'Angelo) type of $\partial D$ at the origin is defined by

$$
\begin{array}{r}
\Delta^{1}(\partial D, 0):=\sup \left\{\frac{\delta_{0}(\partial D, u)}{\delta(u)}, u: \Delta \rightarrow\left(\mathbb{R}^{4}, J\right) J\right. \text {-holomorphic nonconstant, } \\
u(0)=0\} .
\end{array}
$$

The point 0 is a point of finite (D'Angelo) type $2 m$ if $\Delta^{1}(\partial D, 0)=2 m<$ $+\infty$.

(2) The regular type of $\partial D$ at the origin is defined by

$\Delta_{\text {reg }}^{1}(\partial D, 0):=\sup \left\{\delta_{0}(\partial D, u), u: \Delta \rightarrow\left(\mathbb{R}^{4}, J\right) J\right.$-holomorphic,

$$
\left.u(0)=0, d_{0} u \neq 0\right\} .
$$

The type condition as defined in part (1) of Definition 1.4 was introduced by J.-P. D'Angelo [4, who proved that this coincides with the regular type in complex manifolds of dimension two. It was proved in 2 that the (D'Angelo) type and the regular type coincide in four dimensional almost complex manifolds.

In the next proposition, we describe locally the almost complex structure $J$ and the defining function $\rho$ (see 2] for a proof).

Proposition 1.5. Let $D=\{\rho<0\}$ be a smooth domain in $\mathbb{R}^{4}$. Assume that $\rho$ is $J$-plurisubharmonic on a neighborhood of $\bar{D}$ where the structure $J$ is defined on a fixed neighborhood $U$ of $\bar{D}$. We suppose that the origin $0 \in \partial D$ is a point of finite type $2 \mathrm{~m}$. Then there is a local change of coordinates in a neighborhood of the origin such that, in the new coordinates,

$$
\begin{gathered}
J=\left(\begin{array}{cccc}
a_{1} & b_{1} & 0 & 0 \\
c_{1} & -a_{1} & 0 & 0 \\
0 & 0 & a_{2} & b_{2} \\
0 & 0 & c_{2} & -a_{2}
\end{array}\right)=J_{s t}+O\left(\left|z_{2}\right|\right), \\
\rho=\Re e z_{2}+H_{2 m}\left(z_{1}, \overline{z_{1}}\right)+\widetilde{H}\left(z_{1}, z_{2}\right)+O\left(\left|z_{1}\right|^{2 m+1}+\left|z_{2}\right|\left|z_{1}\right|^{m}+\left|z_{2}\right|^{2}\right),
\end{gathered}
$$

where $H_{2 m}$ is a homogeneous polynomial of degree $2 m$, subharmonic which is not harmonic and

$$
\widetilde{H}\left(z_{1}, z_{2}\right)=\Re e \sum_{k=1}^{m-1} \rho_{k} z_{1}^{k} z_{2} .
$$

A crucial tool for the study of pseudoholomorphic curves into pseudoconvex domains of finite type is the local peak $J$-plurisubharmonic functions whose existence was proved in [2].

Theorem 1.6. Let $D=\{\rho<0\}$ be a domain of finite type in an almost complex manifold $(M, J)$ of dimension four. We suppose that $\rho$ is a smooth defining function of $D, J$-plurisubharmonic on a neighborhood of $\bar{D}$. Let $p \in \partial D$ be a boundary point. Then there exist a function $\varphi_{p}$ and a neighborhood $U$ of $p$ such that $\varphi_{p}$ is continuous up to $\bar{D} \cap U$ and satisfies:

(1) $\varphi_{p}$ is $J$-plurisubharmonic on $D \cap U$,

(2) $\varphi_{p}(p)=0$

(3) $\varphi_{p}<0$ on $\bar{D} \cap U \backslash\{p\}$.

Such a function is called a local peak J-plurisubharmonic function at $p$. 
1.4. The Kobayashi pseudometric. The existence of local pseudoholomorphic discs proved in [8] allows one to define the Kobayashi pseudometric $K_{(M, J)}$ for $p \in M$ and $v \in T_{p} M$ :

$$
\begin{aligned}
K_{(M, J)}(p, v):=\inf \left\{\frac{1}{r}>0, u: \Delta \rightarrow(M, J)\right. \text { J-holomorphic, } & \\
& \left.u(0)=p, d_{0} u(\partial / \partial x)=r v\right\} .
\end{aligned}
$$

Let $d_{(M, J)}$ be the integrated pseudodistance of $K_{(M, J)}$ defined by

$$
\begin{aligned}
d_{(M, J)}(p, q):=\inf \left\{\int_{0}^{1} K_{(M, J)}(\gamma(t), \dot{\gamma}(t)) d t\right. & \\
& \gamma:[0,1] \rightarrow M, \gamma(0)=p, \gamma(1)=q\} .
\end{aligned}
$$

We thus define:

Definition 1.7. (1) The manifold $(M, J)$ is Kobayashi hyperbolic if the integrated pseudodistance $d_{(M, J)}$ is a distance.

(2) Let $D \subset M$ be a domain in an almost complex manifold $(M, J)$. A point $p \in \partial D$ is said to be at finite distance from $q \in D$ if there is a sequence of points $q_{j} \in D$ converging to $p$ and whose Kobayashi distances $d_{(D, J)}\left(q_{j}, q\right)$ to $q$ stay bounded. Otherwise we say that the distance is infinite.

\section{Proof of Theorem 0.1}

In order to prove this theorem, we need the two following lemmas, where the dimension assumption is needed to guarantee the existence of a coordinate system where the lines $\left\{z_{1}=c\right\}$ and $\left\{z_{2}=c^{\prime}\right\}$ are almost complex submanifolds.

Lemma 2.1. Let $\Omega$ be an open subset of $\left(\mathbb{R}^{4}, J\right)$ and let $J$ be an almost complex structure satisfying (1.2). Let $K$ be a compact subset of $\Omega$. There exists $\delta>0$ such that: for every $r \in[0,1)$ there exists a positive constant $C>0$ such that if $u=\left(u_{1}, u_{2}\right): \Delta \rightarrow \Omega$ is a J-holomorphic disc with $u(\Delta) \subset K$, then

$$
\left|\nabla u_{1}(\zeta)\right| \leq C \sup _{t \in \Delta}\left|u_{1}(t)-u_{1}(0)\right| \text { and }\left|\nabla u_{2}(\zeta)\right| \leq C \sup _{t \in \Delta}\left|u_{2}(t)-u_{2}(0)\right| \text {, }
$$

if $\sup _{t \in \Delta}\|u(t)-u(0)\| \leq \delta$ and $\zeta \in \Delta_{r}$.

This lemma is an anisotropic version of a result obtained by S. Ivashkovich and J.-P. Rosay in [7.

Proof. Depending on $u(0) \in K$, one can make a linear change of variables such that in the new coordinates $J(u(0))=J_{s t}$. Set $\alpha_{i}:=\sup _{t \in \Delta}\left|u_{i}(t)-u_{i}(0)\right|$ for $i=1,2$ and consider the scaling map $\Lambda$ from $\mathbb{R}^{4}$ into itself defined by $\Lambda\left(z_{1}, z_{2}\right):=$ $\left(\alpha_{1}^{-1}\left(z_{1}-u_{1}(0)\right), \alpha_{2}^{-1}\left(z_{2}-u_{2}(0)\right)\right)$. Notice that the $\Lambda_{*} J$-holomorphic disc $\Lambda \circ u$ satisfies $\Lambda \circ u(\Delta) \subseteq \Delta \times \Delta$. If $\alpha_{1}$ and $\alpha_{2}$ are small enough, then $\Lambda_{*} J$ is close to $J_{s t}$, as the structure $J$ has a diagonal form. It follows from Proposition 2.3.6 in [9] that for $|z| \leq r$ one gets for $i=1,2,\left|\nabla(\Lambda \circ u)_{i}(z)\right| \leq C$ for some positive constant $C$. Hence $\left|\nabla u_{i}(z)\right| \leq C \alpha_{i}$, as desired. 
A straightforward computation leads to this very useful lemma:

Lemma 2.2. Assume that $J$ is an almost complex structure on $\mathbb{R}^{4}$ satisfying (1.2). Then the Levi form of $\Re e z_{2}$ at $z$ and $v=\left(X_{1}, Y_{1}, X_{2}, Y_{2}\right)$ is equal to

$$
\begin{aligned}
\mathcal{L}_{J} \Re e z_{2}(z, v)= & {\left[\left(a_{1}-a_{2}\right)(z) \frac{\partial a_{2}}{\partial x_{1}}(z)-c_{2}(z) \frac{\partial b_{2}}{\partial x_{1}}(z)+c_{1}(z) \frac{\partial a_{2}}{\partial y_{1}}(z)\right] X_{1} X_{2} } \\
+ & {\left[\left(a_{1}+a_{2}\right)(z) \frac{\partial b_{2}}{\partial x_{1}}(z)-b_{2}(z) \frac{\partial a_{2}}{\partial x_{1}}(z)+c_{1}(z) \frac{\partial b_{2}}{\partial y_{1}}(z)\right] X_{1} Y_{2} } \\
+ & {\left[-\left(a_{1}+a_{2}\right)(z) \frac{\partial a_{2}}{\partial y_{1}}(z)+b_{1}(z) \frac{\partial a_{2}}{\partial x_{1}}(z)-c_{2}(z) \frac{\partial b_{2}}{\partial y_{1}}(z)\right] Y_{1} X_{2} } \\
+ & {\left[\left(a_{2}-a_{1}\right)(z) \frac{\partial b_{2}}{\partial y_{1}}(z)+b_{1}(z) \frac{\partial b_{2}}{\partial x_{1}}(z)-b_{2}(z) \frac{\partial a_{2}}{\partial y_{1}}(z)\right] Y_{1} Y_{2} } \\
& +\left[\frac{\partial a_{2}}{\partial y_{2}}(z)-\frac{\partial b_{2}}{\partial x_{2}}(z)\right]\left(c_{2}(z) X_{2}^{2}-2 a_{2}(z) X_{2} Y_{2}-b_{2}(z) Y_{2}^{2}\right) .
\end{aligned}
$$

Proof of Theorem 0.1 . Let $U$ be a neighborhood of 0 in $\mathbb{R}^{4}$. We assume that, on $U$, the structure $J$ satisfies (1.2) and that the defining function has the local expression

$$
\rho=\Re e z_{2}+H_{2 m}\left(z_{1}, \overline{z_{1}}\right)+O\left(\left|z_{1}\right|^{2 m+1}+\left|z_{2}\right|\left|z_{1}\right|^{m}+\left|z_{2}\right|^{2}\right),
$$

where $H_{2 m}$ is a homogeneous polynomial of degree $2 m$, subharmonic which is not harmonic.

Consider for a positive number $\delta>0$, the following anisotropic polydisc:

$$
Q(0, \delta):=\left\{z \in \mathbb{C}^{2},\left|z_{1}\right|<\delta^{\frac{1}{2 m}},\left|z_{2}\right|<\delta\right\} .
$$

Notice that since the defining function $\rho$ satisfies (2.1), for a sufficiently small $\delta<1$, if $z \in Q(0, \delta)$, then we have dist $(z, \partial D) \leq c \delta$ for some positive constant $c>0$.

Let $q^{\prime}=\left(q_{1}^{\prime}, q_{2}^{\prime}\right) \in \partial D \cap U$ be a boundary point and let $\varphi_{q^{\prime}}$ be a local peak $J$-plurisubharmonic function at the point $q^{\prime}$. There is a positive constant $C_{1}$ such that

$$
-C_{1}\left\|z-q^{\prime}\right\| \leq \varphi_{q^{\prime}}(z) \leq-C_{2} \Psi_{q^{\prime}}(z)
$$

where

$$
\Psi_{q^{\prime}}(z):=\left|z_{1}-q_{1}^{\prime}\right|^{2 m}+\left|z_{2}-q_{2}^{\prime}\right|^{2}+\left|z_{1}-q_{1}^{\prime}\right|^{2}\left|z_{2}-q_{2}^{\prime}\right|^{2}
$$

is a $J$-plurisubharmonic function on $U$, shrinking $U$ if necessary.

Let $u: \Delta \rightarrow D \cap U$ be a $J$-holomorphic disc such that $u(0) \in Q(0, \delta)$ is sufficiently close to the origin. In order to prove that the origin 0 is at infinite distance from points in $D \cap U$, we want to provide the following estimates:

$$
\left|\nabla u_{1}(0)\right| \leq C \delta^{\frac{1}{2 m}} \text { and }\left|\nabla u_{2}(0)\right| \leq C \delta
$$

for a positive constant $C>0$.

Let $q^{\prime}=\left(q_{1}^{\prime}, q_{2}^{\prime}\right) \in \partial D$ be the unique boundary point such that $q^{\prime}=u(0)+\left(0, \delta_{u}\right)$ for some positive $\delta_{u}$. Notice that $\delta_{u}$ is asymptotically equivalent to $\operatorname{dist}(u(0), \partial D)$. According to the $J$-plurisubharmonicity of $\Psi_{q^{\prime}}$, we have for $|\zeta|<r$, where $0<r<1$,

$$
\Psi_{q^{\prime}}(u(\zeta)) \leq \frac{A}{2 \pi} \int_{0}^{2 \pi} \Psi_{q^{\prime}}\left(u\left(r e^{i \theta}\right)\right) d \theta
$$


for an appropriate positive constant $A$. Hence using (2.2) and the $J$-plurisubharmonicity of the peak function $\varphi_{q^{\prime}}$ we obtain

$$
\Psi_{q^{\prime}}(u(\zeta)) \leq-\frac{A}{2 \pi C_{2}} \int_{0}^{2 \pi} \varphi_{q^{\prime}}\left(u\left(r e^{i \theta}\right)\right) d \theta \leq-\frac{A}{C_{2}} \varphi_{q^{\prime}}(u(0)) .
$$

Since

$$
\left|u_{2}(\zeta)-q_{2}^{\prime}\right|^{2} \leq \Psi_{q^{\prime}}(u(\zeta))
$$

and according to (2.2) and to the fact that $\left\|u(0)-q^{\prime}\right\|=\delta_{u}$ is asymptotically equivalent to dist $(u(0), \partial D)$, we obtain for a positive constant $C_{3}$ :

$$
\left|u_{2}(\zeta)-q_{2}^{\prime}\right|^{2} \leq C_{3} \operatorname{dist}(u(0), \partial D) .
$$

Hence, for some other positive constant $C_{3}$ we have

$$
\left|u_{2}(\zeta)-u_{2}(0)\right| \leq C_{3} \operatorname{dist}(u(0), \partial D)^{\frac{1}{2}},
$$

for $|\zeta|<r$ where $0<r<1$.

For the same reason, we obtain the following estimate which holds for $|\zeta|<r<1$ :

$$
\left|u_{1}(\zeta)-u_{1}(0)\right| \leq C_{3} \operatorname{dist}(u(0), \partial D)^{\frac{1}{2 m}} .
$$

According to Lemma 2.1, inequalities (2.3) and (2.4) imply

$$
\left|\nabla u_{2}(\zeta)\right| \leq B \sup _{|t|<r}\left|u_{2}(t)-u_{2}(0)\right| \leq C_{3} B \operatorname{dist}(u(0), \partial D)^{\frac{1}{2}} \leq C_{4} \delta^{\frac{1}{2}}
$$

and

$$
\left|\nabla u_{1}(0)\right| \leq B \sup _{|t|<r}\left|u_{1}(t)-u_{1}(0)\right| \leq C_{3} B \operatorname{dist}(u(0), \partial D)^{\frac{1}{2 m}} \leq C_{4} \delta^{\frac{1}{2 m}}
$$

for positive constants $B$ and $C_{4}$. Notice that (2.6) is the desired tangential estimate.

In order to obtain the normal estimate $\left|\nabla u_{2}(0)\right|$, we will construct a negative harmonic function. To achieve this, we first need to control $\Re e u_{2}(\zeta)$ and $\left|\Delta \Re e u_{2}(\zeta)\right|$ by $\delta$.

From (2.3) and (2.4) we obtain for $|\zeta|<r$ :

$$
\left|u_{1}(\zeta)\right| \leq\left|u_{1}(\zeta)-u_{1}(0)\right|+\left|u_{1}(0)\right| \leq C_{3} \operatorname{dist}(u(0), \partial D)^{\frac{1}{2 m}}+\delta^{\frac{1}{2 m}} \leq C_{5} \delta^{\frac{1}{2 m}}
$$

and

$$
\left|u_{2}(\zeta)\right| \leq\left|u_{2}(\zeta)-u_{2}(0)\right|+\left|u_{2}(0)\right| \leq C_{3} \operatorname{dist}(u(0), \partial D)^{\frac{1}{2}}+\delta \leq C_{5} \delta^{\frac{1}{2}}
$$

for a positive constant $C_{5}$. According to these estimates and since the defining function satisfies (2.1), we obtain

$$
\begin{aligned}
\Re e u_{2}(\zeta) & \leq\left|H_{2 m}\left(u_{1}(\zeta), \overline{u_{1}(\zeta)}\right)\right|+O\left(\left|u_{1}(\zeta)\right|^{2 m+1}+\left|u_{2}(\zeta)\right|\left|u_{1}(\zeta)\right|^{m}+\left|u_{2}(\zeta)\right|^{2}\right) \\
& \leq C_{6} \delta .
\end{aligned}
$$

if $|\zeta|<r$, where $C_{6}>0$. Due to Proposition 1.2 applied to the function $\Re e z_{2}$, we have

$$
\Delta \Re e u_{2}(\zeta)=\mathcal{L}_{J} \Re e z_{2}\left(u(\zeta), \frac{\partial u}{\partial x}(\zeta)\right)
$$


Notice that according to (2.8) and to the fact that $J(z)=J_{s t}+O\left(\left|z_{2}\right|\right)$, the partial derivatives of the coefficients of $J$ in $x_{1}$ and $y_{1}$ at $u(\zeta)$, namely $\frac{\partial a_{2}}{\partial x_{1}}(u(\zeta)), \frac{\partial a_{2}}{\partial y_{1}}(u(\zeta))$, $\frac{\partial b_{2}}{\partial x_{1}}(u(\zeta))$ and $\frac{\partial b_{2}}{\partial y_{1}}(u(\zeta))$, are $O\left(\delta^{\frac{1}{2}}\right)$. Then Lemma 2.2 and (2.5) imply

$$
\left|\Delta \Re e u_{2}(\zeta)\right| \leq C_{7} \delta
$$

if $|\zeta|<r$, and a positive constant $C_{7}$.

We still denote by $\Delta \Re e u_{2}$ the function equal to $\Delta \Re e u_{2}$ on $\Delta_{r}$, and 0 elsewhere. Also consider the following harmonic function:

$$
g(\zeta):=\Re e u_{2}(\zeta)-\left[\frac{1}{2 \pi} \ln |\zeta| * \Delta \Re e u_{2}\right](\zeta)-\left(C_{6}+C_{7}\right) \delta .
$$

From (2.11), we have

$$
\left|\frac{1}{2 \pi} \ln \right| \zeta\left|* \Delta \Re e u_{2}\right| \leq C_{7} \delta
$$

Thus $g$ is a negative function and $|g(0)| \leq 2\left(C_{6}+C_{7}\right) \delta$. The classical Schwarz Lemma for negative harmonic functions gives $|\nabla g(0)| \leq 2|g(0)|$. Hence we obtain the following estimate:

$\left|\nabla \Re e u_{2}(0)\right| \leq|\nabla g(0)|+\operatorname{Sup}\left|\Delta \Re e u_{2}\right| \leq 2|g(0)|+C_{7} \delta \leq\left(4 C_{6}+5 C_{7}\right) \delta$.

Moreover, the $J$-holomorphy equation for the disc $u$ implies

$$
\begin{aligned}
& a_{2}(u(0)) \frac{\partial \Re e u_{2}}{\partial x}(0)+b_{2}(u(0)) \frac{\partial \Im m u_{2}}{\partial x}(0)=\frac{\partial \Re e u_{2}}{\partial y}(0) \\
& c_{2}(u(0)) \frac{\partial \Re e u_{2}}{\partial x}(0)-a_{2}(u(0)) \frac{\partial \Im m u_{2}}{\partial x}(0)=\frac{\partial \Im m u_{2}}{\partial y}(0)
\end{aligned}
$$

so that

$$
\left|\nabla \Im m u_{2}(0)\right| \leq C_{8}\left|\nabla \Re e u_{2}(0)\right|,
$$

for some positive constant $C_{8}$.

We finally get the normal estimate:

$$
\left|\nabla u_{2}(0)\right| \leq C \delta .
$$

Let us show how (2.6) and (2.13) imply that the origin 0 is at infinite distance from points in $D \cap U$. Consider the function

$$
\chi(z):=\left|z_{1}\right|^{4 m}+\left|z_{2}\right|^{2} .
$$

Since $u(0) \in Q\left(0, \chi(u(0))^{\frac{1}{2}}\right)$, it follows from the estimates (2.6) and (2.13) that $\left|\nabla u_{1}(0)\right| \leq C \chi(u(0))^{\frac{1}{4 m}}$ and $\left|\nabla u_{2}(0)\right| \leq C \chi(u(0))^{\frac{1}{2}}$ for some positive constant $C$, and thus

$$
\begin{aligned}
|\nabla(\chi \circ u)(0)| & \leq 4 m\left|\nabla u_{1}(0)\right|\left|u_{1}(0)\right|^{4 m-1}+2\left|\nabla u_{2}(0)\right|\left|u_{2}(0)\right| \\
& \leq 4 m C \chi(u(0))^{\frac{1}{4 m}}\left(\chi(u(0))^{\frac{1}{4 m}}\right)^{4 m-1}+2 C \chi(u(0))^{\frac{1}{2}} \chi(u(0))^{\frac{1}{2}} \\
& \leq(4 m C+2 C)(\chi \circ u)(0),
\end{aligned}
$$

which achieves the proof of Theorem 0.1 by an integration argument (see Lemma 1.1 in [7]). 


\section{ACKNOWLEDGMENTS}

The author would like to thank J. Bland and J.-P. Rosay for helpful discussions. He is particularly indebted to J.-P. Rosay, who pointed out an erroneous argument in the previous version of the paper.

\section{REFERENCES}

[1] F. Berteloot, Principe de Bloch et estimations de la métrique de Kobayashi dans les domaines de $\mathbb{C}^{2}$, J. Geom. Anal. 13-1 (2003), 29-37. MR1967034 (2004a:32018)

[2] F. Bertrand, Pseudoconvex regions of finite D'Angelo type in four dimensional almost complex manifolds, Math. Z. 264 (2010), no. 2, 423-459. MR2574983 (2011a:32042)

[3] D. Catlin, Estimates of invariant metrics on pseudoconvex domains of dimension two, Math. Z. 200 (1989), 429-466. MR978601 (90e:32029)

[4] J.-P. D'Angelo, Finite type conditions for real hypersurfaces, J. Diff. Geometry 14 (1979), 59-66. MR.577878 (82a:32022)

[5] H. Gaussier, A. Sukhov, Estimates of the Kobayashi metric on almost complex manifolds, Bull. Soc. Math. France 133 (2005), no. 2, 259-273. MR2172267 (2006f:32036)

[6] I. Graham, Boundary behaviour of the Carathéodory and Kobayashi metrics on strongly pseudoconvex domains in $\mathbb{C}^{n}$ with smooth boundary, Trans. Amer. Math. Soc. 207 (1975), 219-240. MR0372252(51:8468)

[7] S. Ivashkovich, J.-P. Rosay, Schwarz-type lemmas for solutions of $\bar{\partial}$-inequalities and complete hyperbolicity of almost complex manifolds, Ann. Inst. Fourier 54 (2004), no. 7, 2387-2435. MR2139698 (2006a:32032)

[8] A. Nijenhuis, W. Woolf, Some integration problems in almost-complex and complex manifolds, Ann. Math. 77 (1963), 429-484. MR0149505 (26:6992)

[9] J.-C. Sikorav, Some properties of holomorphic curves in almost complex manifolds, Holomorphic Curves in Symplectic Geometry, M. Audin and J. Lafontaine, eds., Birkhäuser (1994), 165-189. MR:1274929

Department of Mathematics, University of Wisconsin, 480 Lincoln Drive, Madison, WisCONSIN 53706

E-mail address: bertrand@math.wisc.edu 